\title{
Factors that Predict Delayed Neurological Sequelae of Carbon Monoxide Poisoning from a 10-Year Clinico-Radiological Review
}

\section{Dear Editor,}

Carbon monoxide (CO) is a highly toxic gas that is formed from incomplete combustion of hydrocarbon compounds. Worldwide, CO poisoning has gained widespread recognition as an important public health problem that presents with deleterious effects in the acute and delayed phases. ${ }^{1,2}$ While acute complications of $\mathrm{CO}$ poisoning are clinically evident, delayed neurological sequelae (DNS) has a broad spectrum of presentations that can mimic other medical conditions. ${ }^{3}$ Greater awareness amongst physicians of delayed sequelae and the features that predict them may justify appropriate initiation of potential treatment such as hyperbaric oxygen therapy (HBOT). Better recognition of DNS during recovery can minimise unnecessary investigations.

In this study, we described the epidemiology of $\mathrm{CO}$ poisoning in Singapore and analysed the clinical and radiological features of patients in the acute and delayed stages. Additionally, we identified features that predict DNS.

\section{Materials and Methods}

A retrospective review of patients who were treated for acute $\mathrm{CO}$ poisoning between 2005-15 in the National Neuroscience Institute - a tertiary neuroscience centre in Singapore - was performed. Patients were identified from hospital records according to the diagnosis codes found in the International Statistical Classification of Diseases and Related Health Problems, $10^{\text {th }}$ revision and contained the terms "carbon monoxide poisoning" and "toxic encephalopathy". Their demographic, clinical (including information on $\mathrm{CO}$ exposure), biochemical (carboxyhaemoglobin level, creatine kinase and white blood cell count), radiological and outcome data were collected. Inclusion criteria included a history of witnessed or suspected exposure to sources of $\mathrm{CO}$ and elevated serum carboxyhaemoglobin ( $>3 \%$ in non-smokers and $>10 \%$ in smokers) at presentation. The study was approved by the Institutional Review Board.
All neuroimaging findings were reviewed by an experienced neuroradiologist and detailed neuroanatomical abnormalities were recorded. Magnetic resonance imaging (MRI) studies comprised at least T2 weighted (T2W), diffusion-weighted and gradient echo (GRE) sequences of the whole brain in all patients. In some patients, fluid-attenuated inversion recovery (FLAIR) and T1-weighted (T1W) images were also available. Abnormal signal (hyperintensity) on T2W/ FLAIR sequences, haemorrhage (susceptibility on GRE and/or T1W hyperintensity) and restricted diffusion (hyperintensity on diffusionweighted images with corresponding low signal on apparent diffusion coefficient map) were documented. MRI studies were classified as "early" when they were performed $\leq 7$ days after presentation, and "late" when they were completed after $>7$ days. DNS was defined as any new neurological, cognitive or affective disorder that developed after an asymptomatic period of any duration following recovery from acute $\mathrm{CO}$ poisoning. 3.5

Statistical analyses were performed using SPSS Statistics for Windows, Version 23 (IBM Corp., Armonk, NY, USA). All variables were summarised with the use of descriptive statistics. Chi-square and Fisher's Exact tests were used to assess associations between categorical variables of interest (clinical symptoms and investigations) and DNS development. Multiple logistic regression was used on predictors that were deemed significant in univariate analysis. A value of $P<0.05$ was considered statistically significant.

\section{Results}

The baseline characteristics of 45 patients included in the study are summarised in Table 1 . Their median age was 38 years (range 19-85 years) and there were more male $(\mathrm{n}=29,64.4 \%)$ patients. Most of the cases $(\mathrm{n}=35,77.8 \%)$ resulted from non-accidental poisoning.

In $21(46.7 \%)$ patients, loss of consciousness was the most common presenting symptom. Five $(11.1 \%)$ patients experienced memory difficulties and $1(2.2 \%)$ each had hypotension and seizures. Concomitant 
Table 1. Clinical Characteristics of Patients with CO Poisoning

\begin{tabular}{|c|c|}
\hline Variable & $N=45$ \\
\hline \multicolumn{2}{|l|}{ Age in years } \\
\hline Mean (SD) & $40(14.2)$ \\
\hline Median (range) & $38(19-85)$ \\
\hline Male gender $(\%)$ & $29(64.4)$ \\
\hline \multicolumn{2}{|l|}{ Medical history (\%) } \\
\hline Psychological disorder & $21(46.7)$ \\
\hline Depression & $16(35.6)$ \\
\hline Previous self-harm/suicide attempt & $5(11.1)$ \\
\hline \multicolumn{2}{|l|}{ Clinical manifestations (\%) } \\
\hline Memory difficulties & $5(11.1)$ \\
\hline Loss of consciousness & $21(46.7)$ \\
\hline \multicolumn{2}{|l|}{ Glasgow Coma Scale $(n=43)$} \\
\hline Mean (SD) & $12.8(3.7)$ \\
\hline Median (range) & $15(3-15)$ \\
\hline$<15(\%)$ & $16(37.2)$ \\
\hline$<9(\%)$ & $7(16.3)$ \\
\hline Seizure $(\%)$ & $1(2.2)$ \\
\hline Hypotension (\%) & $1(2.2)$ \\
\hline Non-accidental poisoning ( $\%)$ & $35(77.8)$ \\
\hline Concomitant BZD use & $8(17.8)$ \\
\hline Concomitant alcohol use & $5(11.1)$ \\
\hline Concomitant BZD and/or alcohol use & $12(26.7)$ \\
\hline \multicolumn{2}{|l|}{ Laboratory results } \\
\hline \multicolumn{2}{|l|}{ Carboxyhaemoglobin $(n=43)$} \\
\hline Mean (SD) & $19.3(14.8)$ \\
\hline$>15 \%(\%)$ & $22(51.2)$ \\
\hline \multicolumn{2}{|l|}{ Creatine kinase $(n=26)$} \\
\hline Mean (SD) & $391.6(551.7)$ \\
\hline$>250 \mathrm{IU} / \mathrm{L}(\%)$ & $9(34.6)$ \\
\hline White blood cell count, mean (SD) & $12.8(5.9)$ \\
\hline \multicolumn{2}{|l|}{ Treatment } \\
\hline Hyperbaric oxygen therapy (\%) & $7(15.6)$ \\
\hline Levodopa $(\%)$ & $7(15.6)$ \\
\hline \multicolumn{2}{|l|}{ Outcomes } \\
\hline Delayed neurological sequelae (\%) & $8(17.8)$ \\
\hline Median time to DNS in days (range) & $16.5(7-40)$ \\
\hline \multicolumn{2}{|l|}{ Length of stay in days } \\
\hline Mean (SD) & $16.5(32.7)$ \\
\hline Median (range) & $5(1-190)$ \\
\hline Discharge to place other than home $(\mathrm{n}=44, \%)$ & $14(31.8)$ \\
\hline Improved after discharge (\%) & $42(93.3)$ \\
\hline
\end{tabular}

benzodiazepines and/or alcohol use was associated with decreased consciousness with a Glasgow Coma Scale (GCS) score of $<15$ at admission (odds ratio [OR] 4.60, 95\% confidence interval [CI] 1.12-18.87, $P=0.041)$. HBOT was administered in $7(15.6 \%)$ patients.

DNS was observed in $8(17.8 \%)$ patients with the common presentation being akinetic mutism; median time to development was 16.5 days (range 7-40 days). Table 2 shows the clinical risk factors associated with the development of DNS. Only low GCS score $(<9)$ on admission was associated with DNS (OR 8.25, 95\% CI 1.23-55.56, $P=0.045$ ), but the finding was not statistically significant after adjusting for age and treatment with HBOT (adjusted OR 7.14, 95\% CI 0.95-53.65, $P=0.056$ ).

Mean hospital stay-including subsequent admissions for DNS-was 16.5 days (standard deviation [SD] 32.7). Most patients were discharged home $(\mathrm{n}=30,68.2 \%)$ or to another health institution for rehabilitation, psychiatric or long-term nursing care $(\mathrm{n}=14,31.2 \%) ; 1$ patient who had multiple comorbidities that included carcinoma of the bladder passed away from pneumonia.

Eleven of the 45 patients underwent initial computed tomography (CT) of the brain. Bilateral globus

Table 2. Clinical Factors Associated with Development of Delayed Neurological Sequelae

\begin{tabular}{lcc}
\hline Factor & $\begin{array}{c}\text { Odds Ratio (95\% } \\
\text { Confidence Interval) }\end{array}$ & P Value \\
\hline Age & $1.05(1.00-1.11)$ & 0.053 \\
\hline Gender (men vs women) & $1.83(0.32-10.31)$ & 0.691 \\
\hline $\begin{array}{l}\text { Depression } \\
\text { Non-accidental vs accidental } \\
\text { carbon monoxide poisoning }\end{array}$ & $3.94(0.80-19.43)$ & 0.111 \\
\hline $\begin{array}{l}\text { Benzodiazepine and/or } \\
\text { alcohol use }\end{array}$ & $0.90(0.16-5.22)$ & 1.000 \\
\hline $\begin{array}{l}\text { Initial memory difficulties } \\
\text { Glasgow Coma Scale on } \\
\text { admission }<9\end{array}$ & $3.78(0.52-27.60)$ & 0.211 \\
\hline $\begin{array}{l}\text { Carboxyhaemoglobin } \\
\text { oxygen therapy }\end{array}$ & $8.25(1.23-55.56)$ & $\mathbf{0 . 0 4 5}$ \\
\hline $\begin{array}{l}\text { Treatment with hyperbaric } \\
\text { As continuous variable }\end{array}$ & $1.00(0.95-1.06)$ & 0.949 \\
\hline $\begin{array}{l}\text { Creatine kinase }>250 \text { IU/L } \\
\text { White blood cell count }\end{array}$ & $8.00(0.69-92.70)$ & 0.104 \\
\hline
\end{tabular}


pallidus hypodensities was found in 3 of them, and all went on to develop DNS (Fig. 1A). In 2 patients with normal CT findings, T2/FLAIR white matter hyperintensities were seen on contemporaneous brain MRI, 1 was performed on the same day and the other, 4 days after CT was done.

During the study period, 13 patients underwent MRI; 10 were done "early" and 3 were done "late". The main indications for MRI were persistently low or deteriorating levels of consciousness in the acute phase and/or development of clinical features of DNS. MRI abnormalities in each patient are described in Table 3.

In the 10 patients who had early MRI, 7 showed T2/ FLAIR hyperintensities in the globus pallidus; 1 of them had concomitant haemorrhage (Fig. 1B). In 3 patients, MRI findings revealed diffuse extensive T2/FLAIR white matter hyperintensities; restricted diffusion was seen in 2 of them.

In the 3 patients who had late MRI, abnormalities were seen in 2 that showed bilateral globus pallidus hyperintensities with haemorrhage. In the remaining patient (Patient 5), extensive T2/FLAIR deep white matter hyperintensities with restricted diffusion was seen on interval scan at day 35 (Fig. 1C-E).

In 8 patients who developed DNS, globus pallidus abnormalities were seen in early MRI of 5 patients and late MRI of 2 patients. Extensive bilateral T2/ FLAIR white matter hyperintensities were seen in 4 patients; in 3 of them, they were seen in early MRI. Restricted diffusion in the white matter was seen in 3 patients, 1 of them in late MRI. In 1 patient (Patient 8), generalised cerebral volume loss was seen on MRI at 5 years (Fig. 1F). All 3 patients with normal early

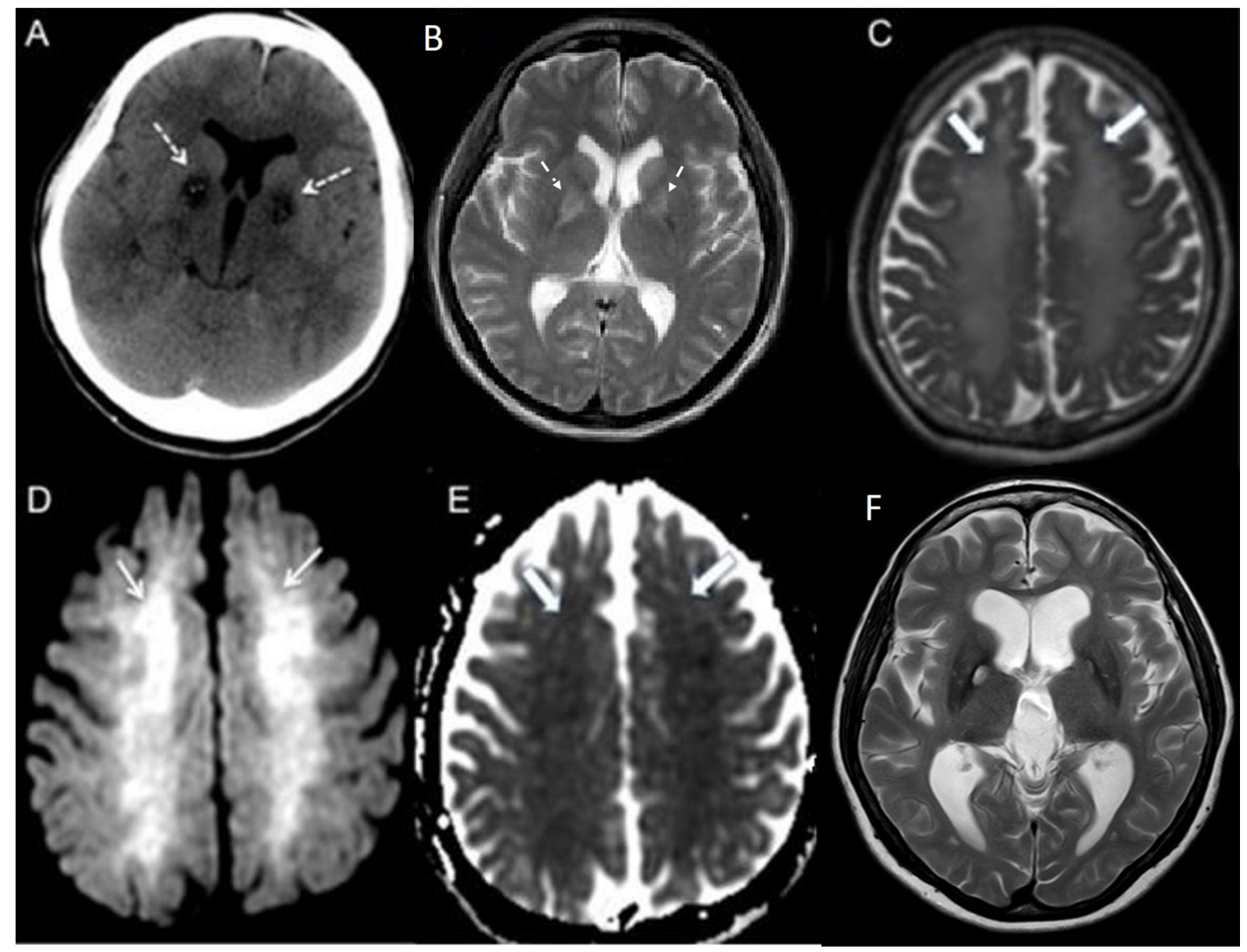

Fig. 1. Neuroimaging in acute and delayed phases of carbon monoxide poisoning. A: At presentation, axial non-contrast computed tomography of brain showed bilateral symmetrical hypodensities in globus pallidus (dashed white arrows). B: On day 7, axial T2W MRI post-exposure showed bilateral hyperintensities in globus pallidus (short dashed white arrows). C: On day 35, axial T2W MRI showed bilateral confluent hyperintensities in cerebral white matter (thick white arrows). D and E: On day 35, ADC showed confluent DWI $\left(\mathrm{b}=1000 \mathrm{~s} / \mathrm{mm}^{3}\right)$ hyperintensities in bilateral centrum semiovale (white arrows) with corresponding low signal on ADC (thick white arrows), indicating restricted diffusion. F: At 5 years postexposure, axial T2W MRI showed widened sulci and increased ventricular dilatation (compared with 1B), indicating volume loss. ADC: Apparent diffusion coefficient; DWI: Diffusion-weighted image; T2W MRI: T2-weighted magnetic resonance image 


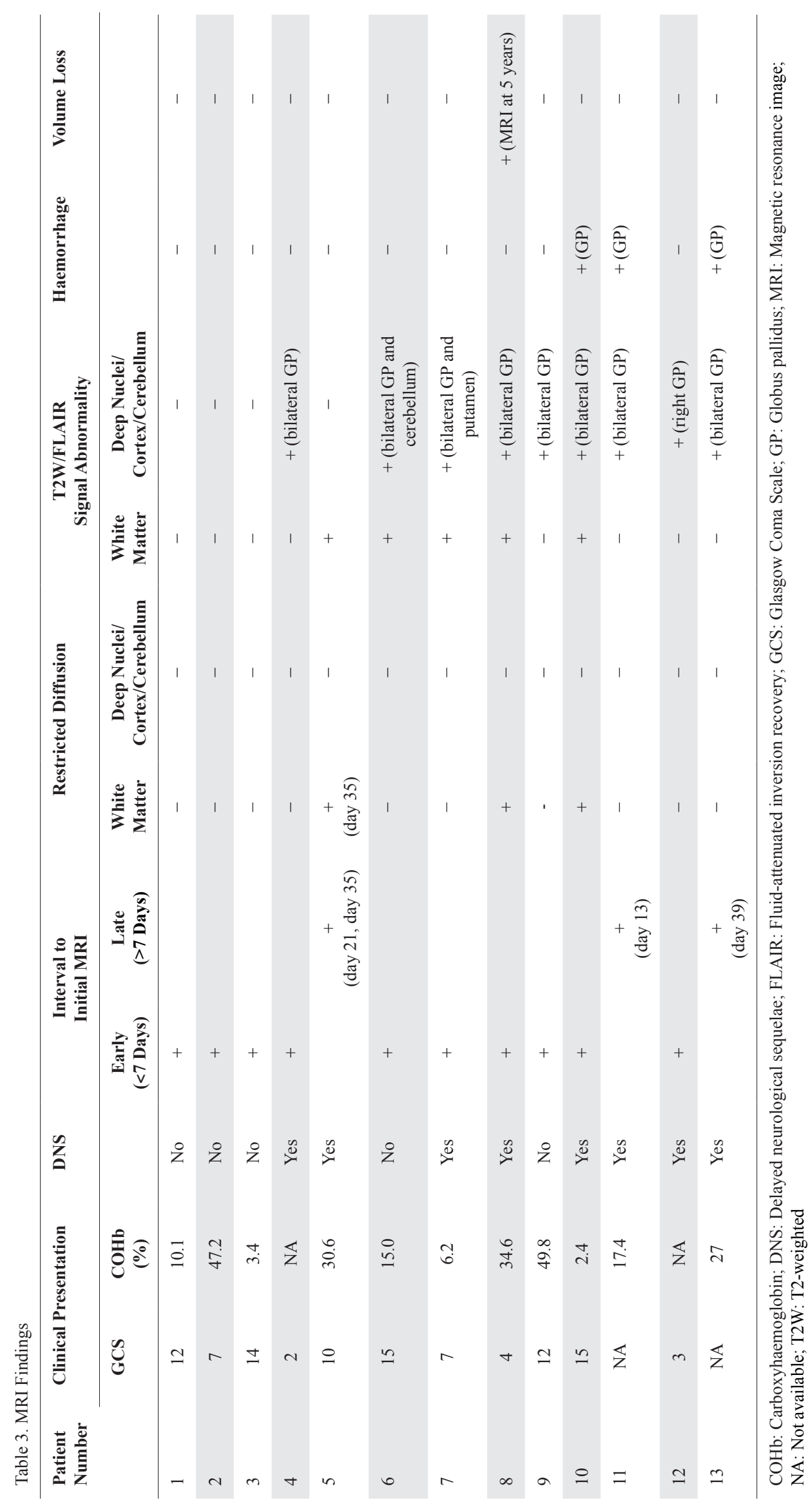


MRI did not develop DNS, while 5 (71.4\%) of 7 patients with abnormal early MRI developed DNS.

\section{Discussion}

In this 10-year review of acute $\mathrm{CO}$ poisoning, no clinical prognostic factors were identified in the development of DNS. Previous studies also did not conclusively identify these factors. In their study, Pepe et al showed that patients aged $\geq 36$ years old who were exposed to $\mathrm{CO}$ for $>24$ hours and did not receive HBOT or had cerebellar abnormalities on presentation had an increased risk of developing cognitive sequelae. ${ }^{3}$ Weaver et al also found that GCS score of $<9$ and leucocytosis at presentation were associated with DNS onset. ${ }^{6}$

On the other hand, radiological investigations that were performed at acute presentation may be helpful to identify features that predict DNS and could potentially guide acute clinical management. In our study, radiological features of $\mathrm{CO}$ poisoning were better demonstrated on MRI. Florid globus pallidus abnormalities could be detected on early CT and may portend the development of neurological complications as all 3 of our patients who had CT-detectable changes developed DNS. In our 8 patients who developed DNS, MRI findings showed a combination of globus pallidus and deep white matter involvement; 3 of them had white matter with restricted diffusion. A recent study concluded that diffusion abnormalities seen on MRI in the acute phase of $\mathrm{CO}$ poisoning was associated with a 14-fold increase in risk of developing DNS. ${ }^{7}$

In our patients, the most common abnormal finding on MRI was bilateral globus pallidus T2 hyperintensities which is consistent with those of previous studies..$^{8-11}$ The vulnerability of the globus pallidus to tissue hypoxia in $\mathrm{CO}$ poisoning is not well understood, but it may be attributed to poor anastomotic blood supply. Another possibility is that due to its high iron content, $\mathrm{CO}$ binds selectively to the globus pallidus which leads to infarction and haemorrhagic conversion. ${ }^{8}$

Extensive confluent T2/FLAIR hyperintensities in the cerebral white matter-with or without restricted diffusion - were also observed in our patients in the acute and delayed phases of presentation. While some authors have postulated that restricted diffusion in the acute phase, which often normalises, is caused by reversible ischaemia, others have attributed it to acute myelinopathy secondary to failure of cellular energy. ${ }^{9,12}$ In the delayed stage, white matter hyperintensities are thought to result from delayed posthypoxic demyelination that is caused by biochemical changes triggered by accumulation of metabolic byproducts. ${ }^{10}$ In a study by Kim et al, this was associated with delayed-onset encephalopathy. ${ }^{13}$
Similar to an earlier local cohort in 2005 who had a mean age of 38.9 years with a preponderance of male (75\%) patients, ${ }^{5}$ most of the patients in our study were young men. However, unlike a 1975 study that found only $9 \%$ of patients presented with non-accidental CO poisoning, in our study the incidence was $77.8 \% .{ }^{14}$ This finding might be attributed to safer workplace practices and declining popularity in the use of gas water heaters. Strict legislation has also curbed the abuse of illicit drugs in the country; consequently, this has led to death by $\mathrm{CO}$ poisoning as an alternative suicide method, a phenomenon that is peculiar to Asian countries. ${ }^{15}$ Since a significant proportion of patients $(60 \%)$ had a history of psychiatric illnesses, managing physicians should consider $\mathrm{CO}$ poisoning in patients with pre-existing psychiatric illnesses who present with a history of unwitnessed loss of consciousness. Additionally, concomitant sedative use should be screened when a patient has a low GCS score at admission.

In our patient cohort, there was significant morbidity with prolonged hospital stay; a third of them also required institutionalisation. DNS occurred in $17.8 \%$ of patients, which was slightly lower than rates (24.1-26.1\%) reported elsewhere. ${ }^{3,7,13}$ This finding could be attributed to the younger mean age ( 38 years old) of our patients than other studies (41-63 years old) that translated to fewer comorbidities.

Although the indications for HBOT remain poorly defined, they have included loss of consciousness, neurological deficits, cardiac ischaemia or pregnancy and, more recently, the finding of restricted diffusion on MRI done in the acute phase. ${ }^{6,7,16}$ In this review, the reasons for the initiation of HBOT were not documented. However, none of the clinical parameters that were examined were associated with HBOT delivery. Additionally, findings of neuroimaging studies were apparently not used to guide clinical care since none of our patients had undergone MRI prior to HBOT. Finally, only 3 of 7 patients who received HBOT had undergone prior brain $\mathrm{CT}$, and only 2 had normal findings. The overall usage of HBOT was low (15.6\%) and the small patient numbers also prevented any meaningful conclusions that can be drawn on the role of HBOT in preventing DNS onset.

Our study has several limitations. Since it was a retrospective review, the data was not complete. Radiological investigations were only performed in a third of patients and comorbidities such as cardiovascular risk factors that could account for white matter hyperintensities on MRI were not captured. However, given the young mean age of our patients, the prevalence of these risk factors is likely to be low. 
Multivariate analysis of clinical risk factors that affected the development of DNS was also limited by the small sample size. Since most of our patients did not undergo a systematic assessment of their functional status or cognitive function, long-term neurological outcomes could not be studied. Nonetheless, to date this is the largest study of CO poisoning in Singapore. It also identified potential areas for improvement in multidisciplinary care of this relatively young and vulnerable group of patients, particularly the appropriate use of early preventive therapies that is guided by neuroimaging studies in the acute phase, better recognition of DNS, more comprehensive neuro-cognitive assessments and targeted rehabilitative strategies.

\section{REFERENCES}

1. Chiew AL, Buckley NA. Carbon monoxide poisoning in the $21 \mathrm{st}$ century. Crit Care 2014;18:221.

2. Raub JA, Mathieu-Nolf M, Hampson NB, Thom SR. Carbon monoxide poisoning - a public health perspective. Toxicology 2000;145:1-14.

3. Pepe G, Castelli M, Nazerian P, Vanni S, Del Panta M, Gambassi F, et al. Delayed neuropsychological sequelae after carbon monoxide poisoning: predictive risk factors in the emergency department. A retrospective study. Scand J Trauma Resusc Emerg Med 2011;19:16.

4. World Health Organization. International Statistical Classification of Diseases and Related Health Problems, 10th Revision. Available at: https://icd.who.int/browse10/2019/en. Accessed on 20 May 2020.

5. Handa PK, Tai DYH. Carbon monoxide poisoning: a five-year review at Tan Tock Seng Hospital, Singapore. Ann Acad Med Singapore 2005;34:611-4.

6. Weaver LK, Hopkins RO, Chan KJ, Churchill S, Elliott CG, Clemmer TP, et al. Hyperbaric oxygen for acute carbon monoxide poisoning. N Engl J Med 2002;347:1057-67.

7. Jeon SB, Sohn CH, Seo DW, Oh BJ, Lim KS, Kang DW, et al. Acute brain lesions on magnetic resonance imaging and delayed neurological sequelae in carbon monoxide poisoning. JAMA Neurol 2018;75:436-43.
8. Lo CP, Chen SY, Lee KW, Chen WL, Chen CY, Hsueh CJ, et al. Brain injury after acute carbon monoxide poisoning: early and late complications. AJR Am J Roentgenol 2007;189:W205-11.

9. Sener RN. Acute carbon monoxide poisoning: diffusion MR imaging findings. AJNR Am J Neuroradiol 2003;24:1475-7.

10. Murata T, Kimura H, Kado H, Omori M, Onizuka J, Takahashi T, et al. Neuronal damage in the interval form of $\mathrm{CO}$ poisoning determined by serial diffusion weighted magnetic resonance imaging plus $1 \mathrm{H}$-magnetic resonance spectroscopy. J Neurol Neurosurg Psychiatry 2001;71:250-3.

11. Lim TCC. Magnetic resonance imaging findings in bilateral basal ganglia lesions. Ann Acad Med Singapore 2009;38:795-8.

12. Chalela JA, Wolf RL, Maldjian JA, Kasner SE. MRI identification of early white matter injury in anoxic-ischemic encephalopathy. Neurology 2001;56:481-5.

13. Kim JH, Chang KH, Song IC, Kim KH, Kwon BJ, Kim HC, et al. Delayed encephalopathy of acute carbon monoxide intoxication: diffusivity of cerebral white matter lesions. AJNR Am J Neuroradiol 2003;24:1592-7.

14. Cheow SH, Cheng CT. Carbon monoxide poisoning in Singapore. Singapore Med J 1975; 16:174-6.

15. Lam SP, Fong SYY, Kwok A, Wong T, Wing YK. Delayed neuropsychiatric impairment after carbon monoxide poisoning from burning charcoal. Hong Kong Med J 2004;10:428-31.

16. Stoller KP. Hyperbaric oxygen and carbon monoxide poisoning: a critical review. Neurol Res 2007;29:146-55.

Shermyn $\underline{\text { Neo, }},{ }^{* 1} M B B S, M R C P$,

Shawn SX $\underline{\mathrm{Kok}},{ }^{* 2}$ MBBS, MMed (Diagnostic Radiology), FRCR,

Kevin $\underline{\text { Tan, }}{ }^{1,3}{ }_{B M} B S, M R C P, M S H P E d$, Sumeet Kumar, ${ }^{4}$ DNB, FRCR, EDiNR

${ }^{1}$ Department of Neurology, National Neuroscience Institute, Singapore

${ }^{2}$ Department of Radiology, Sengkang General Hospital, Singapore

3Duke-NUS Medical School, Singapore

${ }^{4}$ Department of Neuroradiology, National Neuroscience Institute, Singapore

*Both authors contributed equally to the writing of this article.

Address for Correspondence: Dr Shermyn Neo, Department of Neurology, National Neuroscience Institute, 11 Jalan Tan Tock Seng, Singapore 308433. Email: shermyn.neo.x.m@singhealth.com.sg 